\title{
Hopf algebroids and secondary characteristic classes
}

\author{
Jerome Kaminker and Xiang Tang*
}

\begin{abstract}
We study a Hopf algebroid, $\mathscr{H}$, naturally associated to the groupoid $U_{n}^{\delta} \ltimes U_{n}$. We show that classes in the Hopf cyclic cohomology of $\mathscr{H}$ can be used to define secondary characteristic classes of trivialized flat $U_{n}$-bundles. For example, there is a cyclic class which corresponds to the universal transgressed Chern character and which gives rise to the continuous part of the $\rho$-invariant of Atiyah-Patodi-Singer. Moreover, these cyclic classes are shown to extend to pair with the K-theory of the associated $C^{*}$-algebra. This point of view gives leads to homotopy invariance results for certain characteristic numbers. In particular, we define a subgroup of the cohomology of a group analogous to the Gelfand-Fuchs classes described by Connes [3] and show that the higher signatures associated to them are homotopy invariant.
\end{abstract}

Mathematics Subject Classification (2000). 46L87; 58H10.

Keywords. Riemannian foliation, secondary characteristic class, Hopf algebroid, cyclic cohomology, homotopy invariance.

\section{Introduction}

We will study some new cyclic classes which forms a subgroup of the cohomology of a discrete group. In common with the Gelfand-Fuchs classes used by Connes in [3], they extend to pair with the K-theory of a certain algebra and hence yield homotopy invariance results for some "higher signatures". The original goal was to study the Connes-Moscovici index theory for hypoelliptic transverse signature operators, but in the much easier case when the foliation is Riemannian. The spectral triple in that case can be constructed analogous to [5] and one needed to find the appropriate adaptation of the Hopf algebra techniques of [9]. As a step in that direction we considered a very special class of Riemannian-foliated flat $U_{n}$-bundles. We will use a Hopf algebroid to define characteristic classes and relate them to several earlier constructions. The transverse signature operator in this case comes from the signature operator on the compact Lie group $U_{n}$ and its Chern character is in the periodic cyclic cohomology of our Hopf algebroid. However, in the present paper we will look at different aspects of these classes.

\footnotetext{
*The second author thanks NSF for support.
} 
The Hopf algebroid, which we denote $\mathscr{H}\left(U_{n}^{\delta} \ltimes U_{n}\right)$, is one associated to the étale groupoid, $U_{n}^{\delta} \ltimes U_{n}$. We compute its cyclic theory as introduced by Connes and Moscovici [10] and see that it is isomorphic to the cyclic theory of $C_{c}^{\infty}\left(U_{n}^{\delta} \ltimes U_{n}\right)$. Moreover, it has a convenient description via a double complex.

The double complex has a subcomplex corresponding to invariant forms which we investigate further. These classes have two important properties. The first is that the associated cocycles extend to $K .\left(C\left(U_{n}\right) \rtimes U_{n}^{\delta}\right)$, the K-theory of the reduced $\mathrm{C}^{*}$ algebra. This yields homotopy invariance results. The second is that they can be expressed in terms of transgressed classes on the base. A particular case yields the transgressed Chern character, hence yields the homotopy invariance of the (continuous part of) the $\rho$-invariant of Atiyah-Patodi-Singer.

Using the ideas in Connes [3] we also consider the subgroup of the cohomology of a discrete group which is determined by these classes. All of the classes in this subgroup satisfy the Novikov conjecture. It would be interesting to know how much of the cohomology of a group is spanned by these classes and Gelfand-Fuchs classes. This is analogous to results on low-dimensional cohomology of groups and the Novikov conjecture in the sense that the results do not depend on any special properties of the groups.

The authors would like to thank Sasha Gorokhovsky for valuable discussions, and the first author particularly wants to thank Steve Hurder and Ron Douglas since many of the ideas developed here came from his earlier collaboration with them. We also want to thank Alain Connes for suggesting these directions several years ago.

\section{Cyclic cohomology of Hopf algebroids}

In this section we will review Connes and Moscovici's definition, [10], of cyclic cohomology of a Hopf algebroid. We will study a special case related to the classifying space for trivialized flat unitary bundles.

2.1. Hopf algebroids. In [28], Lu introduced the notion of a Hopf algebroid as a generalization of a Hopf algebra. Cyclic cohomology of a Hopf algebroid was developed by Connes and Moscovici, [10], in their study of transverse index theory in the non-flat case.

Let $A$ and $B$ be unital algebras. A bialgebroid structure on $A$ over $B$ consists of the following data:

i) An algebra homomorphism $\alpha: B \rightarrow A$ called the source map and an algebra anti-homomorphism $\beta: B \rightarrow A$ called the target map, satisfying $\alpha(a) \beta(b)=$ $\beta(b) \alpha(a)$ for all $a, b \in A$.

For the next part of the definition let $A \otimes_{B} A$ be the quotient of $A \otimes A$ by the right $A \otimes A$ ideal generated by $\beta(a) \otimes 1-1 \otimes \alpha(a)$ for all $a \in A$. 
ii) A $B$ - $B$ bimodule map $\Delta: A \rightarrow A \otimes_{B} A$, called the coproduct, satisfying

(a) $\Delta(1)=1 \otimes 1$,

(b) $\left(\Delta \otimes_{B}\right.$ id) $\Delta=\left(\right.$ id $\left.\otimes_{B} \Delta\right) \Delta: A \rightarrow A \otimes_{B} A \otimes_{B} A$,

(c) $\Delta(a)(\beta(b) \otimes 1-1 \otimes \alpha(b))=0$ for $a \in A, b \in B$,

(d) $\Delta\left(a_{1} a_{2}\right)=\Delta\left(a_{1}\right) \Delta\left(a_{2}\right)$ for $a_{1}, a_{2} \in A$.

iii) A $B-B$ bimodule map $\epsilon: A \rightarrow B$, called the counit, satisfying

(a) $\epsilon(1)=1$,

(b) $\operatorname{ker} \epsilon$ is a left $A$ ideal,

(c) $\left(\epsilon \otimes_{B}\right.$ id) $\Delta=($ id $\otimes \epsilon) \Delta=$ id: $A \rightarrow A$.

A Hopf algebroid is a bialgebroid $A$ over $B$ which admits an algebra anti-isomorphism $S: A \rightarrow A$ satisfying

(1) $S \circ \beta=\alpha$;

(2) $m_{A}\left(S \otimes\right.$ id) $\Delta=\beta \epsilon S: A \rightarrow A$, with $m_{A}: A \otimes A \rightarrow A$ the multiplication on $A$;

(3) there is a linear map $\gamma: A \otimes_{B} A \rightarrow A \otimes A$ such that

(a) If $\pi: A \otimes A \rightarrow A \otimes_{B} A$ is the natural projection, $\pi \gamma=\mathrm{id}: A \otimes_{B} A \rightarrow$ $A \otimes_{B} A$

(b) $m_{A}($ id $\otimes S) \gamma \Delta=\alpha \epsilon: A \rightarrow A$.

We note that in the above definition one may allow $A$ and $B$ to be differential graded algebras and require all of the above maps to be compatible with the differentials and to be of degree 0 . Thus one would have a differential graded Hopf algebroid. The particular example of a Hopf algebroid which we will study in the present paper is of this type and is inspired by [10], [17].

Example 2.1. We consider a Hausdorff smooth étale groupoid $\mathscr{G} \rightrightarrows G_{0}$. Define $A$ to be the algebra of differential forms ${ }^{1}$ on $\mathcal{H}$, and $B$ to be the algebra of differential forms on $G_{0}$. Both $A$ and $B$ are differential graded (commutative) algebras with the de Rham differential.

We define the source and target map $\alpha, \beta: A \rightarrow B$ as the duals of the source and target maps of the groupoid $\mathcal{G} \rightrightarrows G_{0}$. Similarly, we define $\Delta$ to be the dual of the product on $\mathscr{E} \times{ }_{G_{0}} \mathscr{E} \rightarrow \mathcal{E}$. Note that the algebra of differential forms on $\mathcal{E} \times_{G_{0}} \mathscr{E}$ is equal to $A \otimes_{B} A$. The counit map $\epsilon: A \rightarrow B$ is the embedding $G_{0} \rightarrow \mathscr{G}$ of the unit space. It is straightforward to check that $(A, B, \alpha, \beta, \Delta, \epsilon)$ is a bialgebroid.

The antipode map $S: A \rightarrow A$ is defined to be the dual of the inversion map $\iota: \mathscr{G} \rightarrow \mathcal{E}$. It is easy to check that $S$ satisfies properties (1) and (2) for an antipode of a Hopf algebroid. In the case that $\mathscr{G} \rightrightarrows G_{0}$ is a transformation groupoid from a discrete

\footnotetext{
${ }^{1}$ We remark that we do not require differential forms to be compactly supported.
} 
group $\Gamma$ action on $G_{0}$, we define $\gamma$ to be the dual of the map $\mathcal{E} \times \mathcal{G} \rightarrow \mathcal{E} \times{ }_{G_{0}} \mathcal{E}$ with $\mathcal{E} \times \mathscr{G} \ni\left(\alpha_{1}, m_{1}, \alpha_{2}, m_{2}\right) \mapsto\left(\alpha_{1}, m_{1}, \alpha_{2}, \alpha_{2}^{-1}\left(m_{2}\right)\right) \in \mathscr{E} \times{ }_{G_{0}} \mathscr{E}$. It is not difficult to check that $(A, B, \alpha, \beta, \Delta, \epsilon, S, \gamma)$ is a Hopf algebroid. Since $A$ and $B$ are topological algebras, we want to work in that category and so we will use the projective tensor product. This Hopf algebroid will be referred to as $\mathscr{H}(\mathscr{H})$. In general, we do not know a natural construction of the linear map $\gamma$ required in the above property (3) of an antipode. We refer to [31] for a further discussion about Hopf algebroid associated to a smooth étale groupoid.

2.2. Cyclic cohomology of a Hopf algebroid and Hopf algebroid modules. For completeness, we will review Connes and Moscovici's definition of cyclic cohomology of a Hopf algebroid. The following construction works for a bialgebroid with a twisted antipode $S: A \rightarrow A$. That is, one has an algebra anti-isomorphism such that

$$
S^{2}=\mathrm{id}, \quad S \beta=\alpha, \quad m_{A}\left(S \otimes_{B} \text { id }\right) \Delta=\beta \epsilon S: A \rightarrow A,
$$

and

$$
S\left(a^{(1)}\right)^{(1)} a^{(2)} \otimes_{B} S\left(a^{(1)}\right)^{(2)}=1 \otimes_{B} S(a) .
$$

In the above formula we have used Sweedler's notation for the coproduct $\Delta(a)=$ $a^{(1)} \otimes_{B} a^{(2)}$. We remark that a bialgebroid with this twisted version of an antipode ${ }^{2}$ is called a para-Hopf algebroid in [25].

Proposition 2.2. The bialgebroid $\mathscr{H}(\mathscr{E})=(A, B, \alpha, \beta, \Delta, \epsilon)$ with the antipode $S$ in Example 2.1 for a general étale groupoid forms a para-Hopf algebroid.

Proof. It is sufficient to check equation (2.1). Let $a$ be an element in $A$. For $x, y \in \mathcal{E}$ two composable arrows, we notice that

$$
\Delta(a)(x, y)=a(x y), \quad(S \otimes \mathrm{id}) \Delta(a)(x, y)=a\left(x^{-1} y\right) .
$$

Therefore, one computes

$$
\begin{aligned}
(\Delta S \otimes \mathrm{id}) \Delta(a)(x, y, z) & =a\left(y^{-1} x^{-1} z\right), \\
S\left(a^{(1)}\right)^{(1)} a^{(2)} \otimes_{B} S\left(a^{(1)}\right)^{(2)}(x, y) & =a\left(y^{-1} x^{-1} x\right)=a\left(y^{-1}\right)=1 \otimes_{B} S(a) .
\end{aligned}
$$

Let $\Lambda$ be the cyclic category. We construct a cyclic module $A^{\natural}$ for a para-Hopf algebroid $(A, B, \alpha, \beta, \Delta, \epsilon, S)$.

Define

$$
C^{0}=B, \quad C^{n}=\underbrace{A \otimes_{B} A \otimes_{B} \cdots \otimes_{B} A}_{n}, \quad n \geq 1 .
$$

\footnotetext{
${ }^{2}$ Here in the definition of a twisted antipode, we have fixed a particular modular pair $e=1 \in A$ and $\delta=\epsilon \circ S: A \rightarrow B$. And in general we can extend the following definitions to a Hopf algebroid with a modular pair.
} 
Faces and degeneracy operators are defined as follows:

$\delta_{0}\left(a^{1} \otimes_{B} \cdots \otimes_{B} a^{n-1}\right)=1 \otimes_{B} a^{1} \otimes_{B} \cdots \otimes_{B} a^{n-1}$,

$\delta_{i}\left(a^{1} \otimes_{B} \cdots \otimes_{B} a^{n-1}\right)=a^{1} \otimes_{B} \cdots \otimes_{B} \Delta a^{i} \otimes_{B} \cdots \otimes_{B} a^{n-1}, \quad 1 \leq i \leq n-1$,

$\delta_{n}\left(a^{1} \otimes_{B} \cdots \otimes_{B} a^{n-1}\right)=a^{1} \otimes_{B} \cdots \otimes_{B} a^{n-1} \otimes_{B} 1 ;$

$\sigma_{i}\left(a^{1} \otimes_{B} \cdots \otimes_{B} a^{n+1}\right)=a^{1} \otimes_{B} \cdots \otimes_{B} a^{i} \otimes_{B} \epsilon\left(a^{i+1}\right) \otimes_{B} a^{i+2} \otimes_{B} \cdots \otimes_{B} a^{n+1}$.

The cyclic operators are given by

$$
\tau_{n}\left(a^{1} \otimes_{B} \cdots \otimes_{B} a^{n}\right)=\left(\Delta^{n-1} S\left(a^{1}\right)\right)\left(a^{2} \otimes \ldots a^{n} \otimes 1\right) .
$$

We define the Hopf cyclic cohomology of $A$ to be the cyclic cohomology of $A^{\natural}$. We remark that this definition extends naturally to differential graded Hopf algebroids since all the maps above are maps between differential graded vector spaces and the differential is of degree 1. In the following, we briefly review Gorokhovsky's construction [17] of characteristic class map for a differential graded Hopf algebroid action. We remark that Gorokhovsky only studied characteristic map for a differential graded algebra action. The same construction works for a general para-Hopf algebroid action. In the following we will only state the results and their proofs are actually identical to those in [17].

Let $M$ be a differential graded algebra. Then $M$ is equipped with a Hopf algebroid $(A, B, \alpha, \beta, \Delta, \epsilon, S)$ action if there is a differential graded algebra morphism $\rho: B \rightarrow$ $M$ and an action $\lambda: A \otimes \mathbb{C} M \rightarrow M$ satisfying

(1) $\lambda\left(a_{1} a_{2}, m\right)=\lambda\left(a_{1}, \lambda\left(a_{2}, m\right)\right.$ for $a_{1}, a_{2} \in A, m \in M$;

(2) $\operatorname{deg}(\lambda(a, m))=\operatorname{deg}(a)+\operatorname{deg}(m)$ and

$$
d(\lambda(a, m))=\lambda(d a, m)+(-1)^{\operatorname{deg}(a)} \lambda(a, d m)
$$

for $a \in A, m \in M$;

(3) $\lambda(1, m)=m$ for $m \in M$;

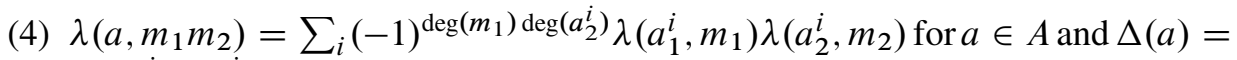
$\sum_{i} a_{1}^{i} \otimes a_{2}^{i} \in A \otimes_{B} A$

and when $M$ has a unit, we require

(5) $\lambda(a, 1)=\lambda(\epsilon(a), 1)=\rho(\epsilon(a))$ for $a \in A$.

We assume that there is a trace $\tau$ on $M$ such that

$$
\tau(\lambda(a, m) n)=(-1)^{\operatorname{deg}(a) \operatorname{deg}(m)} \tau(m \lambda(S(a), n)) \quad \text { for all } m, n \in M \text { and all } a \in A .
$$

We define a cochain map $c$ from the cyclic cochain complex of $A$ to the cyclic cochain complex of the differential graded algebra $M$, i.e., for $\Phi=a_{1} \otimes \cdots \otimes a_{q}$ an element in $C^{q}$ we have $c(\Phi)\left(m_{0}, \ldots, m_{q}\right)=c\left(\Phi, m_{0}, \ldots, m_{q}\right) \tau\left(m_{0} \lambda\left(a_{1}, m_{1}\right) \ldots \lambda\left(a_{q}, m_{q}\right)\right), \quad m_{i} \in M$, 
with $c\left(\Phi, m_{0}, \ldots, m_{1}\right)=(-1)^{\sum_{j=1}^{q} \operatorname{deg}\left(a_{j}\right)\left(\operatorname{deg}\left(m_{j-1}\right)+\cdots+\operatorname{deg}\left(m_{0}\right)\right)}$. We call a cyclic cochain on $M$ in the image of the form $c(\Phi)$ a differentiable cochain, and denote the space of differentiable $p$-cochains by $C_{d}^{p}(M)$. Then we can define differentiable cyclic cohomology of $M, H C_{d}^{*}(M)$, to be the cyclic cohomology of $C_{d}^{\natural}(M)$. One can also define the periodic differentiable cyclic cohomology, $H P_{d}^{*}(M)$, by similar means. One has the following natural map

$$
c: H C^{*}(A) \rightarrow H P_{d}^{*}(M) \rightarrow H P^{*}(M) .
$$

For later applications, we assume that the trace $\tau$ is of weight $q$, i.e., $\tau(m)=0$ if $\operatorname{deg}(m) \neq q$. Then we easily see that a cochain in $A^{\natural}$ with total degree greater than $q$ is mapped to zero by $c$. Let $F^{l} A^{\natural}=\left\{a_{1} \otimes \ldots a_{j} \mid \operatorname{deg}\left(a_{1}\right)+\cdots+\operatorname{deg}\left(a_{j}\right) \geq l\right\}$ and $A_{q}^{\natural}=A^{\natural} / F^{q+1} A^{\natural}$. Then $A_{q}^{\natural}$ is again a cyclic module with cyclic cohomology $H C^{*}(A)_{q}$ and $H P^{*}(A)_{q}$. The characteristic map $c$ induces a map $c: H C^{*}(A)_{q} \rightarrow$ $H P^{*}(M)$.

Let $M^{0}$ be the degree 0 part of $M$. We can map cyclic cochains on $M$ to $M^{0}$. And we have the following characteristic map analogous to [17], Theorem 8:

$$
c: H C^{*}(A)_{q} \rightarrow H P^{*-q}\left(M^{0}\right) .
$$

For an étale groupoid $\mathcal{E}$, we consider the space $M$ of compactly supported differential forms on $\mathcal{E}$ with the convolution product. As $\mathcal{E}$ is an étale groupoid, the unit space $G_{0}$ is embedded in $\mathcal{E}$ as an open submanifold. This embedding map between spaces defines an algebra homomorphism $\rho$ from $B=\Omega^{*}\left(G_{0}\right)$ to $M$. For $a \in A=\Omega^{*}(\mathcal{G})$ and $m \in M=\Omega_{c}^{*}(\mathscr{E})$, define $\lambda(a, m)=a m \in \Omega_{c}^{*}(\mathscr{E})$ by viewing both $a$ and $m$ as elements of $\mathscr{H}(\mathscr{E})$ and using the product on $\mathscr{H}(\mathscr{E})$. It is a direct check that $\rho$ and $\lambda$ define an action of $\mathscr{H}(\mathscr{G})$ on $M$. Furthermore, we assume that there is a $\mathscr{E}$ invariant orientation on $G_{0}$. The integration of differential forms on $G_{0}$ defines a trace $\tau_{\Omega}$ on $M$ of weight $\operatorname{dim}\left(G_{0}\right)$ compatible with the antipode $S$. Hence as $M^{0}=C_{c}^{\infty}(\mathscr{E})$, we have a characteristic map $c_{\Omega}: H C^{*}(\mathcal{H}(\mathscr{E}))_{\operatorname{dim}\left(G_{0}\right)} \rightarrow H P^{*-\operatorname{dim}\left(G_{0}\right)}\left(C_{c}^{\infty}(\mathscr{E})\right)$.

2.3. Hopf cyclic cohomology of the Hopf algebroid $\mathscr{H}(\boldsymbol{E})$. In this section we will compute the Hopf cyclic cohomology of the para-Hopf algebroid $\mathscr{H}(\mathscr{E})$ introduced in Proposition 2.2 for a smooth étale groupoid.

To formulate the results, we will use the following constructions. Let $B \mathscr{G}^{n}=$ $\underbrace{\mathscr{E} \times \times_{G_{0}} \cdots \times \times_{G_{0}}}_{n} \mathscr{E}$, where $\mathscr{E} \times{ }_{G_{0}} \mathscr{E}$ is the fiber product with respect to the maps $t: \mathscr{E} \rightarrow$ $G_{0}$ and $s: \mathcal{G} \rightarrow G_{0}$. This can be given the structure of a simplicial manifold. Let $\Omega^{*}\left(B \mathscr{G}^{*}\right)$ be the double complex of sheaves of differential forms on the simplicial manifold $B \mathscr{E}^{*}$, [14]. Then $\Omega^{*}\left(B \mathscr{E}^{*}\right)$ has a standard cyclic structure (described below). As $\left(\Omega^{*}, d\right)$ is a resolution of $\mathbb{C}$, we denote the corresponding cyclic cohomology of $\Omega^{*}\left(B \mathscr{E}^{*}\right)$ by $H C^{*}(\mathscr{E}, \mathbb{C})$ and $H P^{*}(\mathscr{E}, \mathbb{C})$. One can now state the following result. 


\section{Theorem 2.3.}

$$
H C^{*}(\mathscr{H}(\mathscr{G}))_{\operatorname{dim}\left(G_{0}\right)}=H C^{*}(\mathscr{E} ; \mathbb{C}) \quad \text { and } H P^{*}(\mathscr{H}(\mathcal{E}))_{\operatorname{dim}\left(G_{0}\right)}=H P^{*}(\mathscr{E} ; \mathbb{C}) .
$$

Proof. As a first step one notes that an $m$-cochain on $\mathscr{H}(\mathcal{E})$ of total degree less than or equal to $\operatorname{dim}\left(G_{0}\right)$ can be identified with a smooth section of the sheaf of differential forms on $B \mathscr{G}^{m}, m \geq 0$. This identification respects the cyclic simplicial structures on $\Omega^{*}\left(B \mathscr{E}^{*}\right)$ and $\mathscr{H}(\mathscr{E})^{\natural}$. Indeed, following the notation in [12], where $\left(a \mid g_{1}, \ldots, g_{n}\right)$ with $\left.a \in \Omega^{*}\left(G_{0}\right)\right|_{t\left(g_{1}\right)}, g_{i} \in \mathcal{G}$ and $s\left(g_{i}\right)=t\left(g_{i+1}\right), i=1, \ldots, n-1$, denotes elements of $\Omega^{*}\left(B \mathscr{E}^{*}\right)$, the cyclic simplicial structure on $\Omega^{*}\left(B \mathscr{G}^{*}\right)$ is given by

$$
\begin{aligned}
& \delta_{i}\left(a \mid g_{1}, \ldots, g_{n}\right),= \begin{cases}\left(a g_{1} \mid g_{2}, \ldots, g_{n}\right), & i=0, \\
\left(a \mid g_{1}, \ldots, g_{i} g_{i+1}, \ldots, g_{n}\right), & 1 \leq i \leq k-1, \\
\left(a \mid g_{1}, \ldots, g_{n-1}\right) & i=n,\end{cases} \\
& \sigma_{i}\left(a \mid g_{1}, \ldots, g_{n}\right),=\left(a \mid \ldots, g_{i-1}, 1, g_{i}, \ldots, g_{n}\right),
\end{aligned}
$$

and

$$
t\left(a \mid g_{1}, \ldots, g_{n}\right)=\left(a g_{1} \ldots g_{n} \mid\left(g_{1} g_{2} \ldots g_{n}\right)^{-1}, g_{1}, \ldots, g_{n-1}\right) .
$$

In the above, ag means the right translation of $a$ by $g \in \mathcal{E}$.

It is easy to check from this identification that the $\operatorname{dim}\left(G_{0}\right)$-truncated Hopf cyclic cohomology of $\mathscr{H}(\mathcal{G})$ is equal to the cyclic cohomology of $\Omega^{*}\left(B \mathscr{E}^{*}\right)$.

Just as the cohomology of a discrete group is isomorphic to the cohomology of its classifying space, there is an analogous result for étale groupoids, [29]. It states that the groupoid sheaf cohomology of the sheaf $\mathbb{C}$ on $\mathcal{E}$, denoted $H^{*}(\mathscr{E} ; \mathbb{C})$, is isomorphic to the cohomology of its classifying space $B \mathscr{E}$.

Since $\Omega^{*}$ is a projective resolution of the sheaf $\mathbb{C}$ on $\mathcal{G}$, we obtain the following corollary.

\section{Corollary 2.4.}

$$
\begin{aligned}
& H C^{*}(\mathcal{H}(\mathcal{G}))_{\operatorname{dim}\left(G_{0}\right)}=\bigoplus_{k \in \mathbb{Z}_{+}} H^{*-2 k}(B \mathscr{E} ; \mathbb{C}), \\
& H P^{*}(\mathcal{H}(\mathcal{G}))_{\operatorname{dim}\left(G_{0}\right)}=\bigoplus_{k \in \mathbb{Z}} H^{*+2 k}(B \mathscr{E} ; \mathbb{C}) .
\end{aligned}
$$

We apply Theorem 2.3 in the following special case.

Let $U_{n}$ be the compact Lie group of $n \times n$ unitary matrices and let $U_{n}^{\delta}$ be the same group, but equipped with the discrete topology. Left multiplication of $U_{n}^{\delta}$ on $U_{n}$ defines a transformation groupoid $\mathcal{E}=U_{n}^{\delta} \ltimes U_{n} \rightrightarrows U_{n}$. We will compute the cyclic cohomology of its groupoid algebra, $C_{c}^{\infty}\left(U_{n}^{\delta} \ltimes U_{n}\right)$. The following result was first proved in [1], and we derive it as an application from [12]. 


\section{Proposition 2.5.}

$$
H P^{*}\left(C_{c}^{\infty}\left(U_{n}^{\delta} \ltimes U_{n}\right)\right)=\bigoplus_{k \in \mathbb{Z}} H^{*+2 k+\operatorname{dim}\left(U_{n}\right)}\left(U_{n}^{\delta} \ltimes U_{n}, \mathbb{C}\right) .
$$

Proof. Because $U_{n}^{\delta}$ acts on $U_{n}$ freely, the cyclic groupoid $Z$ introduced in [12] associated to the étale groupoid $U_{n}^{\delta} \ltimes U_{n}$ is equal to itself. Let $o r$ be the orientation sheaf on $U_{n}^{\delta} \ltimes U_{n}$. Then, in [12], 4.14, it is proved that $H P^{*}\left(C_{c}^{\infty}\left(U_{n}^{\delta} \ltimes U_{n}\right)\right)=$ $\bigoplus_{k \in \mathbb{Z}} H^{*+2 k+\operatorname{dim}\left(U_{n}\right)}\left(U_{n}^{\delta} \ltimes U_{n}\right.$, or $)$. Using the left invariant volume form $\Omega$ on $U_{n}$, one can identify the orientation sheaf or and $\mathbb{C}$, and the statement of this proposition follows.

Observe that, in the present situation, the groupoid sheaf cohomology $H^{*}\left(U_{n}^{\delta} \ltimes U_{n}, \mathbb{C}\right)$ is by definition the cohomology of the total complex of

$$
\Gamma\left((\underbrace{U_{n}^{\delta} \times \cdots \times U_{n}^{\delta}}_{q+1}) \times_{U_{n}^{\delta}} U_{n}, \Omega^{p}\left(U_{n}\right)\right),
$$

and the result is equal to the simplicial de Rham cohomology $H^{*}\left(E U_{n}^{\delta} \times_{U_{n}^{\delta}} U_{n}\right)$. We record this fact for later use.

Corollary 2.6. The groupoid sheaf cohomology, $H^{*}\left(U_{n}^{\delta} \ltimes U_{n}, \mathbb{C}\right)$ is isomorphic to the de Rham cohomology of the simplicial manifold $H^{*}\left(E U_{n}^{\delta} \times{ }_{U_{n}^{\delta}} U_{n}\right)$.

Let $\overline{B U}_{n}$ denote the geometric realization of the simplicial manifold $E U_{n}^{\delta} \times{ }_{U_{n}^{\delta}} U_{n}$. Recall a map $\phi: \oplus_{k} H^{*+2 k}\left(\overline{B U}_{n}\right) \rightarrow H P^{*-\operatorname{dim}\left(U_{n}\right)}\left(C_{c}^{\infty}\left(U_{n}^{\delta} \ltimes U_{n}\right)\right)$ defined by Connes. This map actually agrees with

$c: \bigoplus_{k} H^{*+2 k}\left(\overline{B U}_{n}\right)=H P^{*}\left(\mathscr{H}\left(U_{n}^{\delta} \ltimes U_{n}\right)\right)_{\operatorname{dim}\left(U_{n}\right)} \rightarrow H P^{*-\operatorname{dim}\left(U_{n}\right)}\left(C_{c}^{\infty}\left(U_{n}^{\delta} \ltimes U_{n}\right)\right)$

by the same argument as in [17]. Note that the composition of the map $c$ with the identification $e$ : $H P^{*}\left(C_{c}^{\infty}\left(U_{n}^{\delta} \ltimes U_{n}^{\delta}\right)\right)=H P^{*+\operatorname{dim}\left(U_{n}\right)}\left(U_{n}^{\delta} \ltimes U_{n}, \mathbb{C}\right)$ is the identity map on $H^{*}\left(U_{n}^{\delta} \ltimes U_{n}, \mathbb{C}\right)$ since $c$ maps a differential form on $U_{n}^{\delta} \ltimes U_{n}$ to a differential current on $U_{n}^{\delta} \ltimes U_{n}$ to pair with cyclic chains on $C_{c}^{\infty}\left(U_{n}^{\delta} \ltimes U_{n}\right)$ and $e$ maps vice versa. In summary, we have obtained the following result.

\section{Corollary 2.7. The map}

$\phi: \bigoplus_{k} H^{*+2 k}\left(\overline{B U}_{n}\right)=H P^{*}\left(\mathcal{H}\left(U_{n}^{\delta} \ltimes U_{n}\right)\right)_{\operatorname{dim}\left(U_{n}\right)} \rightarrow H P^{*-\operatorname{dim}\left(U_{n}\right)}\left(C_{c}^{\infty}\left(U_{n}^{\delta} \ltimes U_{n}\right)\right)$

is an isomorphism. 
C. Lazarov and J. Pasternack have introduced secondary characteristic classes for Riemannian foliations with trivial normal bundle. We will relate our constructions to theirs, [26]. Let $\overline{B R \Gamma}_{q}$ denote the classifying space for Riemannian Haefliger structures of codimension $q$ with trivial normal bundle. We remark that the dimension of $U_{n}$ is $n^{2}$.

Proposition 2.8. There is a canonical Riemannian Haefliger structure on $E U_{n}^{\delta} \times{ }_{U_{n}^{\delta}} U_{n}$ with trivial normal bundle which induces a map $E U_{n}^{\delta} \times_{U_{n}^{\delta}} U_{n} \rightarrow \overline{B R}_{n^{2}}$.

Proof. For the existence of a Riemannian Haefliger structure cover $E U_{n}^{\delta}$ by small open sets $V_{\alpha}$ which are disjoint from all their translates. Choose an invariant Riemannian metric on $U_{n}$ and let $\left\{W_{\beta}\right\}$ be a cover by charts. Then $\operatorname{pr}_{1}: V_{\alpha} \times W_{\beta} \rightarrow \mathbb{R}^{n^{2}}$ descends to the quotient by $U_{n}^{\delta}$ and provides the Haefliger structure. We must show that its normal bundle is trivial. Note first that the tangent bundle $T U_{n}$ is a trivialized by using left translation. Then $\operatorname{pr}_{1}^{*}\left(T U_{n}\right)$ is trivial. Because $U_{n}^{\delta}$ acts trivially on $T U_{n}$, the quotient $\widetilde{T U}_{n}$ of the bundle $p_{1}^{*} T U_{n}$ on $E U_{n}^{\delta} \times_{U_{n}^{\delta}} U_{n}$ is again trivial. But the coordinate transformations for $\widetilde{T U}_{n}$ are obtained from the differentials of those associated to the Haefliger structure, so the conclusion holds.

By [26], there is a characteristic class map obtained as the following composition:

$$
\kappa: H^{*}\left(R W_{n^{2}}\right) \rightarrow H^{*}\left(\overline{B R}_{n^{2}}\right) \rightarrow H^{*}\left(E U_{n}^{\delta} \times_{U_{n}^{\delta}} U_{n}\right)
$$

\section{Secondary characteristic classes of trivialized flat $U_{n}$-bundles}

Let $M$ be a compact smooth manifold and let $\Gamma=\pi_{1}(M)$ be the fundamental group of $M$. Let $\tilde{M}$ be the universal cover of $M$. Suppose that we are given a finite dimensional unitary representation $\alpha: \Gamma \rightarrow U_{n}$. Consider the flat principal $U_{n}$-bundle $V=\tilde{M} \times_{\Gamma} U_{n} \rightarrow M$. If we assume that this bundle is trivial with a given trivialization $\theta: \tilde{M} \times_{\Gamma} U_{n} \rightarrow M \times U_{n}$ then we can relate it to the Lazarov-Pasternack map.

In this section we will construct a map $\chi: H C^{*}\left(\mathscr{H}\left(U_{n}^{\delta} \ltimes U_{n}\right)\right)_{n^{2}} \rightarrow H^{*}(V)$. It will be a composition of several maps.

First, the homomorphism of algebras $C_{c}^{\infty}\left(\Gamma \ltimes U_{n}\right) \rightarrow C_{c}^{\infty}\left(U_{n}^{\delta} \ltimes U_{n}\right)$ induces a homomorphism on cyclic cohomology

$$
v: H P^{*}\left(C_{c}^{\infty}\left(U_{n}^{\delta} \ltimes U_{n}\right)\right) \rightarrow H P^{*}\left(C_{c}^{\infty}\left(\Gamma \ltimes U_{n}\right)\right) .
$$

Next, note that the manifold $V$ is foliated by leaves which are the images of $\tilde{M} \times\{g\}$. We denote this foliation by $\mathcal{F}$, and the corresponding holonomy groupoid by $\mathcal{G}$. We assume that the representation $\alpha$ is faithful, which will assure that the 
holonomy groupoid $\mathscr{E}$ is Hausdorff. Furthermore, $\mathscr{E}$ is Morita equivalent to the transformation groupoid $\Gamma \ltimes U_{n}$. Therefore the groupoid algebra $C_{c}^{\infty}(\mathscr{G})$ is Morita equivalent to $C_{c}^{\infty}\left(\Gamma \ltimes U_{n}\right)$. Thus, there is an isomorphism on cyclic cohomology, which provides the second map

$$
\iota: H P^{*}\left(C_{c}^{\infty}\left(\Gamma \ltimes U_{n}\right)\right) \rightarrow H P^{*}\left(C_{c}^{\infty}(\mathscr{E})\right) .
$$

Recall that in [4], Chapter III, 7. $\gamma$, Connes constructs a map $\lambda: H P^{*}\left(C_{c}^{\infty}(\mathscr{E})\right) \rightarrow$ $H^{*+n^{2}}(B \mathscr{E})$ which is a left inverse to the map $\Phi$ which is important in higher index theorems. Finally, the foliated structure on $V$ defines a map $V \rightarrow B \mathscr{G}$. The induced map on cohomology will be denoted $\eta: H^{*}(B \mathscr{E}) \rightarrow H^{*}(V)$.

We summarize the above constructions in the following sequence

$$
\begin{gathered}
H C^{*}\left(\mathcal{H}\left(U_{n}^{\delta} \ltimes U_{n}\right)\right)_{n^{2}} \stackrel{c}{\rightarrow} H P^{*-n^{2}}\left(C_{c}^{\infty}\left(U_{n}^{\delta} \ltimes U_{n}\right)\right) \stackrel{\nu}{\rightarrow} H P^{*-n^{2}}\left(C_{c}^{\infty}\left(\Gamma \ltimes U_{n}\right)\right) \\
\stackrel{\iota}{\rightarrow} H P^{*-n^{2}}\left(C_{c}^{\infty}(\mathcal{G})\right) \stackrel{\lambda}{\rightarrow} \bigoplus_{k} H^{*+2 k}(B \mathcal{G}) \stackrel{\eta}{\rightarrow} \bigoplus_{k} H^{*+2 k}(V) .
\end{gathered}
$$

We define

$$
\chi: H C^{*}\left(\mathscr{H}\left(U_{n}^{\delta} \ltimes U_{n}\right)\right)_{n^{2}} \rightarrow \bigoplus_{k} H^{*+2 k}(V)
$$

to be the above composition and

$$
\hat{\chi}: H C^{*}\left(\mathscr{H}\left(U_{n}^{\delta} \ltimes U_{n}\right)\right)_{n^{2}} \rightarrow H P^{*-n^{2}}\left(C_{c}^{\infty}\left(\Gamma \ltimes U_{n}\right)\right)
$$

to be the composition of the first two arrows. We will refer to $\chi$ as the characteristic map and cohomology classes in the image of $\chi$ will be viewed as secondary characteristic classes.

These classes are compatible with those introduced by Lazarov and Pasternack as the next proposition shows.

Proposition 3.1. Let $\kappa$ and $\tilde{\kappa}$ be the maps obtained using Lazarov-Pasternack [26], (2.2). Then the following diagram commutes:

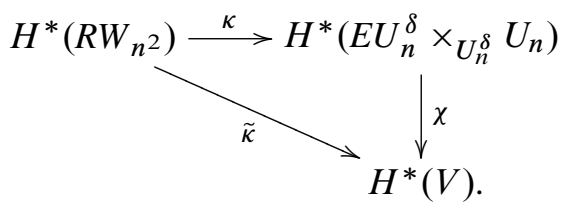

Proof. It is straightforward check that the diagram is commutative.

In the remainder of this section we provide a more detailed description of the map

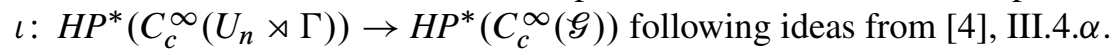


Consider the holonomy groupoid $\mathcal{G} \rightrightarrows V$. The space $\mathcal{E}$ consists of holonomy classes of paths connecting two points on a leaf. The set of homotopy classes of paths on a single leaf is equal to $\widetilde{M} \times \tilde{M}$. Since the leaves are all simply connected, it follows from this that the holonomy groupoid $\mathscr{E}$ can be identified with $(\tilde{M} \times \tilde{M}) \times_{\Gamma} U_{n}$. The structure maps $s, t: \mathcal{E}=(\tilde{M} \times \tilde{M}) \times_{\Gamma} U_{n} \rightrightarrows V=\tilde{M} \times_{\Gamma} U_{n}$ and groupoid operation are defined as follows:

$$
\begin{array}{ll}
s\langle\tilde{x}, \tilde{y}, g\rangle=\langle\tilde{x}, g\rangle, \quad t\langle\tilde{x}, \tilde{y}, g\rangle=\langle\tilde{y}, g\rangle, \quad \tilde{x}, \tilde{y} \in \tilde{M}, g \in U_{n}, \\
\langle\tilde{x}, \tilde{y}, g\rangle \circ\langle\tilde{y}, \tilde{z}, g\rangle=\langle\tilde{x}, \tilde{z}, g\rangle, \quad \tilde{x}, \tilde{y}, \tilde{z} \in \tilde{M} .
\end{array}
$$

Using this description of $\mathcal{G}$, we can make the following identifications.

Let $\mathcal{R}$ be the algebra of infinite matrices $\left(a_{i j}\right)_{i, n \in \mathbb{N}}$ with rapid decay property, i.e.,

$$
\sup _{i, j \in \mathbb{N}} i^{k} j^{l}\left|a_{i j}\right|<\infty \quad \text { for all } k, l \in \mathbb{N} \text {. }
$$

One defines the map

$$
T: C_{c}^{\infty}(\mathcal{G}) \rightarrow M_{N}\left(C_{c}^{\infty}\left(\Gamma \ltimes U_{n}\right) \otimes \mathcal{R}\right),
$$

where $M_{N}\left(C_{c}^{\infty}\left(\Gamma \ltimes U_{n}\right) \otimes \mathcal{R}\right)$ is the algebra of $(N \times N)$-matrices with entries in $C_{c}^{\infty}\left(\Gamma \ltimes U_{n}\right) \otimes \mathcal{R}$.

Since $M$ is a compact manifold, we can choose a finite open cover $\left(U_{i}\right)_{i=1, \ldots, N}$, with $\beta_{i}: U_{i} \rightarrow \tilde{M}$ a local smooth section of the projection $\tilde{M} \rightarrow M$ and $\left(\varphi_{i}\right)_{i=1, \ldots, N}$ a smooth partition of unity subordinate to the covering $\left(U_{i}\right)_{i=1, \ldots, N}$, with $\varphi_{i}^{1 / 2}$ also smooth functions.

Let $\mathcal{R}_{M}$ denote the algebra of smoothing operators on $M$. For any $f \in C_{c}^{\infty}(\mathcal{Y})$, define $T(f) \in M_{N}\left(C_{c}^{\infty}\left(\Gamma \ltimes U_{n}\right) \otimes \mathcal{R}_{M}\right)$, where $\mathcal{R}_{M}$ is the algebra of smoothing operators on $C^{\infty}(M)$ by

$$
T(f)_{i, j}(x, y, \gamma, g)=\varphi_{i}^{\frac{1}{2}}(x) \varphi_{j}^{\frac{1}{2}}(y) f\left(\beta_{i}(x), \gamma^{-1} \beta_{j}(y), g\right),
$$

for $x, y \in M, g \in U_{n}, \gamma \in \Gamma$.

It is straightforward to check that, since $f$ is smooth and compactly supported on $\mathcal{E}, T(g)_{i j}(\gamma, g)$ defines a smoothing operator on $M$ with finite support on $\Gamma$. Similar to [4], III.4. $\beta, T$ is an algebra homomorphism and induces a Morita equivalence between $C_{c}^{\infty}(\mathcal{G})$ and $C_{c}^{\infty}\left(\Gamma \ltimes U_{n}\right)$.

We choose an isomorphism $\varrho$ between $\mathcal{R}_{M}$ and $\mathcal{R}$, which always exists and is unique up to inner automorphisms. The composition $I=\varrho \circ T$ defines an algebra homomorphism from $C_{c}^{\infty}(\mathcal{G})$ to $M_{n}\left(C_{c}^{\infty}\left(\Gamma \ltimes U_{n}\right) \otimes \mathcal{R}\right)$.

We can use $I$ to define a map $\iota: H P^{*}\left(C_{c}^{\infty}\left(\Gamma \ltimes U_{n}\right)\right) \rightarrow H P^{*}\left(C_{c}^{\infty}(\mathscr{G})\right)$ as follows. For $\Psi \in H P^{j}\left(C_{c}^{\infty}\left(\Gamma \ltimes U_{n}\right)\right)$, let

$$
\iota(\Psi)\left(a_{0}, \ldots, a_{j}\right)=\Psi\left(I\left(a_{0}\right)_{i_{0} i_{1}}^{m_{0} m_{1}}, I\left(a_{1}\right)_{i_{1} i_{2}}^{m_{1} m_{2}}, \ldots, I\left(a_{n}\right)_{i_{n} i_{0}}^{m_{n} m_{0}}\right),
$$


where $i_{0}, \ldots, i_{n}$ run from 1 to $\infty$, and $m_{0}, \ldots, m_{n}$ runs from 1 to $N$. This construction can be viewed as the sharp product on cyclic cohomology between $\Psi$ with the standard traces on $M_{n}$ and $\mathcal{R}$.

\section{Extendability of cocycles}

In this section we will describe a subgroup of the cohomology of $\mathscr{H}\left(U_{n}^{\delta} \ltimes U_{n}\right)$, whose image under $\chi$ consists of classes extending to pair with the K-theory of the reduced $\mathrm{C}^{*}$-algebra of $\Gamma \ltimes U_{n}$. The proof is a direct adaptation of Connes' argument in [3].

To describe the subset, consider the double complex $e^{p, q}:=\left(\Omega^{p}\left(U_{n}\right) \times{ }_{U_{n}^{\delta}}\right.$ $\left.\left(U_{n}^{\delta}\right)^{\times(q+1)},(d, b)\right)$ whose total cohomology is $H^{*}\left(U_{n}^{\delta} \ltimes U_{n}\right)$. By [1], the spectral sequence of this double complex degenerates at $E_{2}=H^{q}\left(B U_{n}^{\delta}, H^{p}\left(U_{n}\right)\right)$. We consider a subcomplex $\left(\Omega^{*}\left(U_{n}\right)^{U_{n}}, d\right)=\Omega^{*}\left(U_{n}\right) \times_{U_{n}^{\delta}} U_{n}^{\delta}=\ell^{*, 0} \hookrightarrow \ell^{p, q}$. It is easy to check that the inclusion is a cochain map and therefore defines a homomorphism $H^{*}\left(U_{n}\right) \hookrightarrow H^{0}\left(B U_{n}^{\delta}, H^{*}\left(U_{n}\right)\right) \subseteq H C^{*}\left(\mathscr{H}\left(U_{n}^{\delta} \ltimes U_{n}\right)\right)_{n^{2}}$.

Theorem 4.1. The linear functionals on $K_{*}\left(C_{c}^{\infty}\left(\Gamma \ltimes U_{n}\right)\right)$ defined by elements in $\hat{\chi}\left(H^{*}\left(U_{n}\right)\right) \subset H P^{*-n^{2}}\left(C_{c}^{\infty}\left(U_{n}^{\delta} \ltimes U_{n}\right)\right)$ extend to linear functionals on $K_{*}\left(C\left(U_{n}\right) \rtimes \Gamma\right)$, where $C\left(U_{n}\right) \rtimes \Gamma$ is the reduced crossed product $C^{*}$-algebra.

Proof. We consider the following sequence of maps

$$
\begin{aligned}
H^{*}\left(U_{n}\right) \rightarrow H C^{*} & \left(\mathscr{H}\left(U_{n}^{\delta} \ltimes U_{n}\right)\right)_{n^{2}} \\
& \rightarrow H P^{*-n^{2}}\left(C_{c}^{\infty}\left(U_{n}^{\delta} \ltimes U_{n}\right)\right) \rightarrow H P^{*-n^{2}}\left(C_{c}^{\infty}\left(\Gamma \ltimes U_{n}\right)\right) .
\end{aligned}
$$

Let $\left.\omega \in \Omega^{*}\left(U_{n}\right)\right)^{U_{n}}$ be a representative of a cocycle in $H^{*}\left(U_{n}\right)$ of degree $p$. Notice that the real dimension of $U_{n}$ is $n^{2}$. Then $\chi(\omega) \in H C^{n^{2}-p}\left(C_{c}^{\infty}\left(\Gamma \ltimes U_{n}\right)\right)$ is defined as

$$
\chi(\omega)\left(f_{0}, f_{1}, \ldots, f_{n^{2}-p}\right)=\left.\int_{U_{n}} \omega \wedge\left(f_{0} d f_{1} \ldots d f_{n^{2}-k}\right)\right|_{\mathrm{id}},
$$

where $f_{0}, \ldots, f_{n^{2}-p}$ are elements in $C_{c}^{\infty}\left(\Gamma \ltimes U_{n}\right), d$ is the de Rham differential on $\Omega^{*}\left(U_{n}\right) \rtimes \Gamma$, and $\left.(\ldots)\right|_{\text {id }}$ stands for the restriction of an element in $\Omega^{*}\left(U_{n}\right) \rtimes \Gamma$ to the identity component.

In the following we prove that $\chi(\omega)$ defines an $\left(n^{2}-p\right)$-trace on the reduced $\mathrm{C}^{*}$-algebra $C\left(U_{n}\right) \rtimes \Gamma$ and hence the extension exists. Our proof is essentially the same as Connes' arguments in [3]. However, it simplifies greatly in that we have an isometric (rather than almost isometric) action at hand. In this case the initial step of finding a Banach subalgebra $B$ on which to construct an $n$-trace is unnecessary, since 
the map $\lambda: C_{c}^{\infty}\left(\Gamma \ltimes U_{n}\right) \rightarrow \operatorname{End}_{A}(\mathcal{E})$ is a *-homomorphism, hence is bounded. Thus one can take $B=C\left(U_{n}\right) \rtimes \Gamma$ with the reduced $\mathrm{C}^{*}$-norm.

What we will show next is that elements in the image $\hat{\chi}\left(H^{*}\left(U_{n}\right)\right)$ are $n$-traces which by [3], Theorem 2.7, will define linear maps from $K_{*}(A)$ to $\mathbb{C}$.

We recall the definition of an $n$-trace on a Banach algebra $A$. An $n$-trace on $A$ is an $(n+1)$-linear functional $\tau$ on a dense subalgebra $\mathcal{A}$ of $A$ such that

(1) $\tau$ is a cyclic cocycle on $\mathcal{A}$;

(2) for any $a^{i} \in \mathcal{A}, i=1, \ldots, n$, there exists $C=C_{a^{1}, \ldots, a^{n}}<\infty$ such that

$$
\left|\hat{\tau}\left(\left(x^{1} d a^{1}\right)\left(x^{2} d a^{2}\right) \ldots\left(x^{n} d a^{n}\right)\right)\right| \leq C\left\|x^{1}\right\| \ldots\left\|x^{n}\right\|, \quad x^{i} \in A .
$$

For our purpose, we consider the Banach algebra to be $C\left(U_{n}\right) \rtimes \Gamma$. Given any $\omega \in$ $\Omega^{*}\left(U_{n}\right)^{U_{n}}$ with $d \omega=0$, we have that $\chi(\omega)$ defines a cyclic cocycle on $C_{c}^{\infty}\left(\Gamma \ltimes U_{n}\right)$. To prove that $\chi(\omega)$ defines an $m$-trace, it suffices to prove the estimate (4.1) for any $a^{i} \in \mathcal{A}$ and $x^{i} \in A, i=1, \ldots, n$.

Define on the collection of spaces $\bigoplus_{j} \mathcal{E}_{j}=\bigoplus_{j} C_{c}\left(\Gamma \ltimes U_{n}, r^{*}\left(\wedge^{j} T_{\mathbb{C}}^{*} U_{n}\right)\right)$ a convolution product $*: \mathcal{E}_{i} \otimes \mathcal{E}_{j} \rightarrow \mathcal{E}_{i+j}$ by

$$
\phi * \psi(\alpha)=\sum_{\alpha=\beta \gamma} \phi(\beta) \wedge \beta(\psi(\gamma)), \quad \alpha \in \Gamma \ltimes U_{n} .
$$

Assume that $\omega \in \Omega^{p}\left(U_{n}\right)^{U_{n}}$ with $d \omega=0$. Then $\operatorname{tr}_{\omega}(\phi)=\int_{U_{n}} \omega(x) \phi(x$,id $)$ defines a linear functional on $\mathcal{E}_{n^{2}-p}$ with the following property:

$$
\operatorname{tr}_{\omega}(\phi * \psi)=\operatorname{tr}_{\omega}\left((-1)^{j k} \psi * \phi\right), \quad \psi \in \mathcal{E}_{j}, \phi \in \mathcal{E}_{k}, j+k=n^{2}-p .
$$

The proof of the above trace property goes as follows.

$$
\begin{aligned}
\operatorname{tr}_{\omega}(\phi * \psi) & =\int_{U_{n}} \omega(x) \sum_{\alpha \beta=\mathrm{id}} \phi(x, \alpha) \wedge \psi(\alpha(x), \beta) \\
& =\int_{U_{n}} \omega(x)(-1)^{j k} \sum_{\alpha \beta=\mathrm{id}} \psi(\alpha(x), \beta) \wedge \phi(x, \alpha) \\
& =\int_{U_{n}} \omega\left(\alpha^{-1}(y)\right)(-1)^{j k} \sum_{\alpha \beta=\mathrm{id}} \psi(y, \beta) \wedge \phi(\beta(y), \alpha) \\
& =\int_{U_{n}} \omega(y)(-1)^{j k} \sum_{\alpha \beta=\mathrm{id}} \psi(y, \beta) \wedge \phi(\beta(y), \alpha) \\
& =(-1)^{j k} \operatorname{tr}_{\omega}(\psi * \phi) .
\end{aligned}
$$

In the above proof, $y$ is $\alpha(x)$ and we have used that $\alpha^{-1}=\beta$, and $\omega$ is $\Gamma$ invariant.

We prove the following properties for $\operatorname{tr}_{\omega}$, which is an analog of [3], Lemma 4.3. 
Lemma 4.2. (1) For any $\phi \in \mathcal{E}_{n^{2}-p}$, there is a constant $C_{\phi}<\infty$ such that for any $f \in C\left(U_{n}\right) \rtimes \Gamma$, one has

$$
\left|\operatorname{tr}_{\omega}(f \phi)\right| \leq C_{\phi}\|f\|_{A},
$$

where $\|f\|_{A}$ is the $C^{*}$-algebra norm of the reduced crossed product $C^{*}$-algebra $C\left(U_{n}\right) \rtimes \Gamma$.

(2) For and $\phi_{1}, \ldots, \phi_{n^{2}-p} \in \mathcal{E}_{1}$, there exists a constant $C_{\phi_{1}, \ldots, \phi_{n^{2}-p}}<\infty$ such that for any $f_{1}, \ldots, f_{n} \in C\left(U_{n}\right) \rtimes \Gamma$,

$$
\operatorname{tr}_{\omega}\left(\phi_{1} f_{1} * \phi_{2} f_{2} * \cdots * \phi_{n^{2}-p} f_{n^{2}-p}\right) \leq C_{\phi_{1}, \ldots, \phi_{n^{2}-p}} \prod_{i}\left\|f_{i}\right\| .
$$

Proof. (1) We recall that for any $f \in C\left(U_{n}\right) \rtimes \Gamma$, the following estimates of the $C^{*}$-norm of $\|f\|_{A}$ holds, i.e., $\sup _{x \in U_{n}, \gamma \in \Gamma}|f(x, \gamma)| \leq\|f\|_{A}$. Now for $\operatorname{tr}_{\omega}(f \phi)$, we have that

$$
\begin{aligned}
\left|\operatorname{tr}_{\omega}(f \phi)\right| & =\left|\int_{U_{n}} \sum_{\alpha \beta=\mathrm{id}} \omega(x) f(x, \alpha) \alpha(\phi(\alpha(x), \beta))\right| \\
& \leq \int_{u_{n}}|\omega(x)| \sum_{\alpha \beta=\mathrm{id}}|f(x, \alpha)||\alpha(\phi(\alpha(x), \beta))| \\
& \leq\|f\|_{A} \int_{U_{n}}|\omega(x)| \sum_{\alpha}\left|\alpha\left(\phi\left(\alpha(x), \alpha^{-1}\right)\right)\right| \leq C_{\phi}\|f\|_{A} .
\end{aligned}
$$

We remark that $\sum_{\alpha}\left|\alpha\left(\phi\left(\alpha(x), \alpha^{-1}\right)\right)\right|$ is a finite sum, and therefore the integral is finite.

(2) We apply Connes [3], Theorem 3.7, to the following situation.

Theorem. Let $\Psi$ be an m-linear function on $\mathcal{E}_{1}$ satisfying the following conditions:

(a) $\Psi\left(\xi_{1}, \ldots, \xi_{j} f, \xi_{j+1}, \ldots, \xi_{m}\right)=\Psi\left(\xi_{1}, \ldots, f \xi_{j+1}, \ldots, \xi_{m}\right)$ for $j=1, \ldots$, $m-1, \xi_{k} \in \mathcal{E}_{1}$ and $f \in C\left(U_{n}\right) \rtimes \Gamma$.

(b) For any $\xi_{1}, \ldots, \xi_{m} \in \mathcal{E}_{1}$, there exists $C<\infty$ such that

$$
\left|\Psi\left(\xi_{1}, \ldots, \xi_{m} f\right)\right| \leq C\|f\|_{A}, \quad f \in C\left(U_{n}\right) \rtimes \Gamma .
$$

Then for any $f_{0} \in C\left(U_{n}\right) \rtimes \Gamma$ and $\xi_{1}, \ldots, \xi_{m} \in \mathcal{E}_{1}$, there exists $C^{\prime}<\infty$ with

$$
\left|\Psi\left(f_{0} f_{1} \xi_{1}, f_{2} \xi_{2}, \ldots, f_{m} \xi_{m}\right)\right| \leq C^{\prime}\left\|f_{1}\right\| \ldots\left\|f_{m}\right\|, \quad f_{i} \in C\left(U_{n}\right) \rtimes \Gamma .
$$

To apply the above theorem to our situation, we introduce $\Psi_{\omega}$ an $n^{2}-p$ linear functional $\varepsilon_{1}$ as follows. For $\xi_{1}, \ldots, \xi_{n^{2}-p} \in \mathcal{E}_{1}$, let

$$
\Psi_{\omega}\left(\xi_{1}, \ldots, \xi_{n^{2}-p}\right)=\left.\int_{U_{n}} \omega(x) \wedge\left(\xi_{1} * \cdots * \xi_{n^{1}-p}\right)\right|_{\mathrm{id}} .
$$


It is easy to see that $\Psi_{\omega}$ satisfies condition (a) of the above theorem since $\xi_{i} f *$ $\xi_{i+1}=\xi_{i} * f * \xi_{i+1}=\xi_{i} * f \xi_{i+1}$ for any $i=1, \ldots, n^{2}-p-1$. For condition (b), we consider that $\Psi\left(\xi_{1}, \ldots, \xi_{n^{2}-p} f\right)=\operatorname{tr}_{\omega}\left(\xi_{1} * \cdots * \xi_{n^{2}-p} * f\right)=\operatorname{tr}_{\omega}\left(f \xi_{1} * \cdots * \xi_{n^{2}-p}\right)$. Since all $\xi_{i}$ belong to $\varepsilon_{1}, \xi_{1} * \cdots * \xi_{n^{2}-p}$ again belongs to $\varepsilon_{n^{2}-p}$. Hence, by Lemma $3.2(1),\left|\Psi_{\omega}\left(\xi_{1}, \ldots, \xi_{n^{2}-p} f\right)\right|=\left|\operatorname{tr}_{\omega}\left(f \xi_{1} * \cdots * \xi_{n^{2}-p}\right)\right| \leq C_{\xi_{1}, \ldots, \xi_{n^{2}-p}}\|f\|_{A}$. Therefore both condition (a) and (b) are satisfied, and the above theorem implies that

$$
\left|\Psi_{\omega}\left(f_{0} f_{1} \xi_{1}, f_{2} \xi_{2}, \ldots, f_{n^{2}-p} \xi_{n^{2}-p}\right)\right| \leq C^{\prime}\left\|f_{1}\right\| \ldots\left\|f_{n^{2}-p}\right\|
$$

Now using the tracial property, we have

$$
\begin{aligned}
\left|\operatorname{tr}_{\omega}\left(\phi_{1} f_{1} * \ldots \phi_{n^{2}-p} f_{n^{2}-p}\right)\right| & =\left|\operatorname{tr}_{\omega}\left(f_{n^{2}-p} \phi_{1} * f_{1} \phi_{2} * \ldots * f_{n^{2}-p-1} \phi_{n^{2}-p}\right)\right| \\
& =\left|\Psi_{\omega}\left(f_{n^{2}-p} \phi_{1}, \ldots, f_{n^{2}-p-1} \phi_{n^{2}-p}\right)\right| \\
& \leq C^{\prime}\left\|f_{1}\right\| \ldots\left\|f_{n^{2}-p}\right\| .
\end{aligned}
$$

This proves Lemma 4.2.

Now by Lemma 4.2 and the tracial property, we can easily obtain the condition (4.1), and we conclude that $\hat{\chi}(\omega) \in H C^{n^{2}-p}\left(C_{c}^{\infty}\left(\Gamma \ltimes U_{n}\right)\right)$ defines a linear function from $K_{*}\left(C\left(U_{n}\right) \rtimes \Gamma\right)$ to $\mathbb{C}$ for any $\omega \in \Omega^{p}\left(U_{n}\right)^{U_{n}}$ with $d \omega=0$.

Remark 4.3. We can extend $U_{n}$ in Theorem 4.1 to a general oriented manifold $V$ with a vector bundle $E$ which is equipped with an almost isometric orientation-preserving action of $\Gamma$. Then the similar statements hold for the closed $\Gamma$ invariant differential forms on $V$. In [3], Connes applies this idea to fundamental cocycles. In the context, one can see that he definitely has this kind of generalization in mind.

Remark 4.4. We remark that Jiang [19] proved that if a discrete group $\Gamma$ is rapid decay and acts isometrically on a closed oriented Riemannian manifold $V$ preserving the orientation, then cocycles on $E \Gamma \times_{\Gamma} V$ of polynomial growth can be paired with the K-theory group of the reduced $C^{*}$-algebra $C_{0}(V) \rtimes \Gamma$. (See [19] for the precise statement.) Our results in this section show that any $\Gamma$-invariant cocycles on $V$ can be paired with the K-theory of the $\mathrm{C}^{*}$-algebra for any orientation-preserving isometrical action of a discrete group $\Gamma$ on an oriented Riemannian manifold. We do not need to assume the group to be rapid decay.

\section{Transgressed classes and cyclic classes}

The goal of the present section is to study the transgressed Chern character of a flat trivialized $U_{n}$-bundle. By providing a simplicial construction of the universal transgressed Chern character we will be able to show that it is in the image of the 
map $\chi$. Indeed, the proof actually shows that any class in $\chi\left(H^{*}\left(U_{n}\right)\right)$ is obtained by transgression from an invariant polynomial applied to curvature forms.

We start from the following fibration

$$
\overline{B U}_{n} \rightarrow B U_{n}^{\delta} \rightarrow B U_{n},
$$

where $\overline{B U}_{n}$ is the homotopy fiber of the map from $B U_{n}^{\delta} \rightarrow B U_{n}$. We can take $\overline{B U}_{n}$ to be the realization of a simplicial space, $E U_{n}^{\delta} \times_{U_{n}^{\delta}} U_{n}$. Now, one knows that $B U_{n}$ is the classifying space for $U_{n}$-bundles, $B U_{n}^{\delta}$ is the classifying space for flat $U_{n}$-bundles, and $\overline{B U}_{n}$ is the classifying space for trivialized flat $U_{n}$-bundles. In particular, we will see that on $E U_{n}^{\delta} \times{ }_{U_{n}^{\delta}} U_{n}$ there is a flat $U_{n}$-bundle equipped with a canonical trivialization.

Consider the universal flat $U_{n}^{\delta}$-bundle over $B U_{n}^{\delta}$, which can be identified with $E U_{n}^{\delta}$. Therefore, the associated flat principal $U_{n}$-bundle over $B U_{n}^{\delta}$ can be identified with $E U_{n}^{\delta} \times_{U_{n}^{\delta}} U_{n} \rightarrow B U_{n}^{\delta}$. Considering the map of classifying space, we have the following diagram

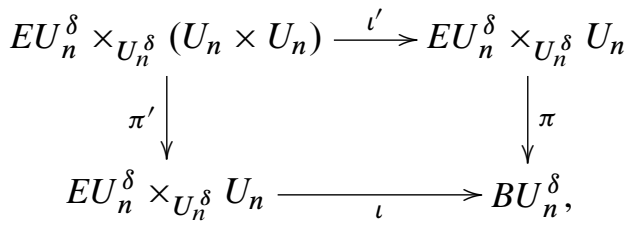

where $E U_{n}^{\delta} \times_{U_{n}^{\delta}}\left(U_{n} \times U_{n}\right)$ with $U_{n}^{\delta}$ acting on $U_{n} \times U_{n}$ diagonally is the pullback with respect to the maps $\iota$ and $\pi$.

We write the maps $\pi, \pi^{\prime}, \iota, \iota^{\prime}$ in coordinates. We write a point in $E U_{n}^{\delta} \times_{U_{n}^{\delta}} U_{n}$ by $(z, x)$ with $z \in E U_{n}^{\delta}$ and $x \in U_{n}$. Then $\pi(z, x)=\iota(z, x)=[z]$, where $[z]$ stands for the $U_{n}^{\delta}$ orbit of $z$ in $E U_{n}^{\delta}$. And $(z, x, y)$ with $z \in E U_{n}^{\delta}, x, y \in U_{n}$ form local $U_{n}^{\delta}$-equivariant coordinates for $E U_{n}^{\delta} \times{ }_{U_{n}^{\delta}}\left(U_{n} \times U_{n}\right)$ such that $\iota^{\prime}(z, x, y)=(z, y)$ and $\pi^{\prime}(z, x, y)=(z, x)$.

On the bundle $\pi^{\prime}: E U_{n}^{\delta} \times_{U_{n}^{\delta}}\left(U_{n} \times U_{n}\right) \rightarrow E U_{n}^{\delta} \times_{U_{n}^{\delta}} U_{n}$ there are two natural connections. One is the pull back of the flat connection on $\pi: E U_{n}^{\delta} \times_{U_{n}^{\delta}} U_{n} \rightarrow B U_{n}^{\delta}$, the other is from a canonical trivialization $E U_{n}^{\delta} \times_{U_{n}^{\delta}}\left(U_{n} \times U_{n}\right) \rightarrow\left(E U_{n}^{\delta} \times_{U_{n}^{\delta}} U_{n}\right) \times U_{n}$.

(1) The flat connection on $\pi: E U_{n}^{\delta} \times_{U_{n}^{\delta}} U_{n} \rightarrow B U_{n}^{\delta}$ is given by Dupont [14] in a simplicial way. We consider the universal $U_{n}^{\delta}$-bundle $\pi: \overline{N U}_{n}^{\delta} \rightarrow N U_{n}^{\delta} . \overline{N U}_{n}^{\delta}$ is the simplicial manifold with $\overline{N U}_{n}^{\delta}(k)=U_{n}^{\delta} \times \cdots \times U_{n}^{\delta}(k+1$ copies $)$, where the face operators are defined by leaving out one of the components. Similarly, $N U_{n}^{\delta}$ is simplicial manifold with $N U_{n}^{\delta}(k)=U_{n}^{\delta} \times \cdots \times U_{n}^{\delta}$ ( $k$-copies) with the face operator equal to the multiplication of consecutive two components. $E U_{n}^{\delta}$ and $B U_{n}^{\delta}$ are the corresponding geometrical realizations of $\overline{N U}_{n}^{\delta}$ and $N U_{n}^{\delta}$. There is 
a canonical map from $\overline{N U}_{n}^{\delta} \rightarrow N U_{n}^{\delta}$ defined by

$$
\pi\left(x_{0}, \ldots, x_{k}\right)=\left(x_{0} x_{1}^{-1}, x_{1} x_{2}^{-1}, \ldots, x_{k-1} x_{k}^{-1}\right) .
$$

We consider the maps $\operatorname{pr}_{i}: \overline{N U}_{n}^{\delta} \times_{U_{n}^{\delta}} U_{n} \rightarrow U_{n}$ defined by

$$
\operatorname{pr}_{i}\left(x_{0}, \ldots, x_{k} ; x\right)=x_{i} x \text {. }
$$

Furthermore, let $\theta$ be the Maurer-Cartan form on $U_{n}$ and $\left(t^{0}, \ldots, t^{k}\right)$ be the barycentric coordinates of the simplex $\Delta_{k}$. Then $\Theta=\sum_{i} t^{i} \operatorname{pr}_{i}^{*}(\theta)$ defines a connection 1-form on $E U_{n}^{\delta} \times_{U_{n}^{\delta}} U_{n} \rightarrow B U_{n}^{\delta}$. The pullback of this connection through $\iota^{\prime}$ defines a connection 1-form on $E U_{n}^{\delta} \times{ }_{U_{n}^{\delta}}\left(U_{n} \times U_{n}\right)$, which is denoted by $\Theta_{0}=\iota^{\prime *}(\Theta)$.

(2) We construct a canonical trivialization of $E U_{n}^{\delta} \times_{U_{n}^{\delta}}\left(U_{n} \times U_{n}\right) \rightarrow E U_{n}^{\delta} \times{ }_{U_{n}^{\delta}} U_{n}$ by using the natural section $\sigma: E U_{n}^{\delta} \times{ }_{U_{n}^{\delta}} U_{n} \rightarrow E U_{n}^{\delta} \times_{U_{n}^{\delta}}\left(U_{n} \times U_{n}\right)$ given by $\sigma(z, x)=(z, x, x)$. Accordingly we have a map $\Sigma: E U_{n}^{\delta} \times{ }_{U_{n}^{\delta}}\left(U_{n} \times U_{n}\right) \rightarrow U_{n}$ defined by $\Sigma(z, x, y)=y^{-1} x$. If $\theta$ is the Maurer-Cartan form on $U_{n}$, then $\Sigma^{*}(\theta)$ defines a connection 1-form on $E U_{n}^{\delta} \times_{U_{n}^{\delta}}\left(U_{n} \times U_{n}\right)$ which is denoted by $\Theta_{1}=\Sigma^{*}(\theta)$ and which is associated to the section $\sigma$.

We consider the following connection $D=s \Theta_{0}+(1-s) \Theta_{1}$ on the space $\left(E U_{n}^{\delta} \times_{U_{n}^{\delta}}\left(U_{n} \times U_{n}\right)\right) \times[0,1]$, where $s$ is the coordinate on the interval [0,1]. Let $R_{s}$ be the curvature of $D$. Let $\mathfrak{U}_{n}$ be the Lie algebra of $U_{n}$. For any $\mathcal{P} \in \operatorname{sym}\left(\mathfrak{U}_{n}^{*}\right)^{\mathfrak{u}_{n}}$, we define $T \mathcal{P} \in \Omega^{*}\left(E U_{n}^{\delta} \times_{U_{n}^{\delta}}\left(U_{n} \times U_{n}\right)\right)$ by

$$
T \mathcal{P}=\int_{0}^{1} \mathcal{P}\left(R_{S}\right) .
$$

Using the expression $D=s \Theta_{0}+(1-s) \Theta_{1}$, we have that

$$
R_{s}=d s \wedge \Theta_{0}+s d \Theta_{0}-d s \wedge \Theta_{1}+(1-s) d \Theta_{1}+\frac{1}{2} s^{2} \Theta_{0}^{2}+(1-s) s \Theta_{0} \wedge \Theta_{1}+\frac{1}{2}(1-s)^{2} \Theta_{1}^{2} .
$$

It is easy to check that $R_{S}$ is $\mathfrak{U}_{n}$ horizontal, and therefore descends to a 2-form on $\left(E U_{n}^{\delta} \times_{U_{n}^{\delta}} U_{n}\right) \times[0,1]$. Consider the projection map $\pi^{\prime}: E U_{n}^{\delta} \times_{U_{n}^{\delta}}\left(U_{n} \times U_{n}\right) \times[0,1] \rightarrow$ $E U_{n}^{\delta} \times{ }_{U_{n}^{\delta}} U_{n} \times[0,1]$, which forgets the second $U_{n}$-factor. We observe that the restrictions of $\Theta_{0}=\sum_{i} t^{i} \operatorname{pr}_{i}^{*}(\theta)$ and $d \Theta_{0}=\sum_{i} d t^{i} \wedge \operatorname{pr}_{i}^{*}(\theta)+t^{i} \operatorname{pr}_{i}^{*}(d \theta)$ to $U_{n}^{\delta^{\times(q+1)}}$ (with $q \geq 0$ ) vanish as $U_{n}^{\delta}$ is equipped with discrete topology, and any differential form with a positive degree on a discrete set always vanishes. We conclude that restrictions of those terms in $R_{S}$ that contain the forms $\Theta_{0}$ or $d \Theta_{0}$ vanish on the base $E U_{n}^{\delta} \times_{U_{n}^{\delta}} U_{n} \times[0,1]$. Thus $R_{S}$ simplifies on $E U_{n}^{\delta} \times_{U_{n}^{\delta}} U_{n} \times[0,1]$ to

$$
-d s \wedge \Theta_{1}+(1-s) d \Theta_{1}+\frac{1}{2}(1-s)^{2} \Theta_{1}^{2} .
$$


For example, when we apply this to the polynomial $\mathcal{P}(X)=\operatorname{Tr}\left(\exp \left(-\frac{1}{2 \pi} X\right)\right) \in$ $\operatorname{sym}\left(\mathcal{U}_{n}^{*}\right)^{\mathfrak{U}_{n}}$, we obtain a formula for the character:

$$
\operatorname{Tch}=\int_{0}^{1} \operatorname{Tr}\left(\exp \left(R_{s}\right)\right)=\int_{0}^{1} d s \sum_{k} \frac{1}{k !} \frac{(-1)^{k+1}}{(2 \pi)^{k+1}} \operatorname{Tr}\left(\Theta_{1}\left(s d \Theta_{1}+\frac{1}{2} s^{2} \Theta_{1}^{2}\right)^{k}\right) .
$$

Consider next $\Theta_{1}$ and $d \Theta_{1}$. These are simplicial differential forms on the components of the simplicial manifold $E U_{n}^{\delta} \times{ }_{U_{n}^{\delta}}\left(U_{n} \times U_{n}\right)(k)=\left(\Delta_{k} \times \overline{N U}_{n}^{\delta}(k)\right) \times{ }_{U_{n}^{\delta}}$ $\left(U_{n} \times U_{n}\right)$ independent of the coordinates on $\Delta_{k}$.

We now consider the mapping from the space of differential forms $\Omega^{l}\left(\left(\Delta_{k} \times\right.\right.$ $\left.\left.\overline{N U}(k)_{n}^{\delta}\right) \times_{U_{n}^{\delta}} U_{n}\right)$ on the simplicial manifold $E U_{n}^{\delta} \times_{U_{n}^{\delta}} U_{n}$ to the group cochain complex $C^{k}\left(U_{n}^{\delta} ; \Omega^{l-k}\left(U_{n}\right)\right)$ of $\Omega^{*}\left(U_{n}\right)$-valued $U_{n}^{\delta}$-cochains. The map $I$ is realized by integration along the $\Delta_{k}$ component of $\left(\Delta_{k} \times \overline{N U}_{n}^{\delta}\right) \times_{U_{n}^{\delta}} U_{n}$. Observing that $T \mathcal{P}$ is independent of the $\Delta_{k}$ coordinates, we conclude that $I(T \mathscr{P})$ gives rise to a zero-dimensional $U_{n}^{\delta}$ cocycle with values in $\Omega^{*}\left(U_{n}\right)^{U_{n}}$.

Finally, we will relate characteristic numbers obtained from these classes to secondary classes for trivialized flat bundles. Note that we can view these constructions in the context of the following diagram.

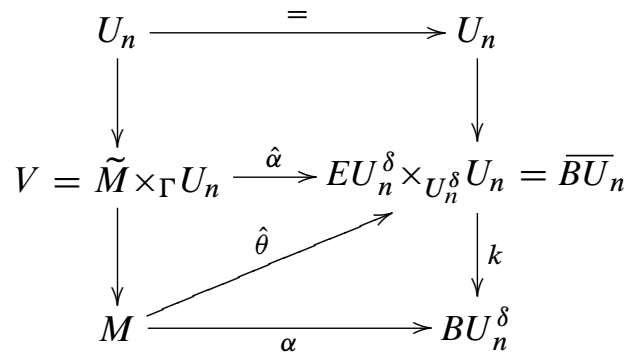

The bundle that was considered in (5.1) is the pull-back of the left side to its total space. As a trivialized flat bundle it is classified by the map $\hat{\alpha}$. The universal class $T \mathcal{P}$ is in $H^{*}\left(\overline{B U}_{n}\right)$. If we assume in addition the existence of a trivialization $\theta: \tilde{M} \times{ }_{\Gamma} U_{n} \rightarrow M \times U_{n}$, then there is a lift of $\alpha$ determined by $\theta$ which we will denote by $\hat{\theta}: M \rightarrow \overline{B U}_{n}$. The pull back of the universal class by $\hat{\theta}$ we will denote by

$$
\hat{\theta}^{*}(T \mathcal{P})=T \mathscr{P}(\alpha, \theta)
$$

This will play a role in the index theorems of the next section.

Combining this with Theorem 4.1 we obtain the following result.

Proposition 5.1. The following holds.

(1) Let $[c] \in \chi\left(H^{*}\left(U_{n}\right)\right)$. Then there exist $i \geq 1$ and polynomials $\mathcal{P}_{1}, \ldots, \mathcal{P}_{i} \in$ $\operatorname{sym}\left(\mathfrak{U}_{n}^{*}\right)^{\mathfrak{U}_{n}}$ such that $[c]=\hat{\alpha}^{*}\left(\left[T \mathcal{P}_{1}\right] \ldots\left[T \mathcal{P}_{i}\right]\right)$. 
(2) Let $\mathcal{P} \in \operatorname{sym}\left(\mathfrak{U}_{n}^{*}\right)^{\mathfrak{U}_{n}}$. Then the associated transgressed class $\hat{\alpha}^{*}(T \mathscr{P})$ is equal to $\chi([c])$ for some cyclic class $[c]$.

(3) The cyclic class from (2) extends to define a linear map on the K-theory of the reduced crossed-product $C^{*}$-algebra:

$$
[c]: K_{*}\left(C\left(U_{n}\right) \rtimes \Gamma\right) \rightarrow \mathbb{C} .
$$

\section{Higher index theorems and secondary classes}

In this section we will apply Connes' index theorem [4] to obtain explicit formulas for pairing our cyclic classes with index classes in K-theory. This will lead in the next section to what appears to be a new family of classes in the cohomology of a group which will satisfy the Novikov conjecture. They are the analog of the Gelfand-Fuchs classes which Connes considered in [3].

Recall the geometric setting as described in Section 2. Let $M$ be a closed $\operatorname{Spin}^{c}$ manifold and let $\alpha: \Gamma=\pi_{1}(M) \rightarrow U_{n}$ be an injective homomorphism. Consider the associated flat, foliated, principal $U_{n}$-bundle $V=\tilde{M} \times_{\alpha} U_{n} \rightarrow M$. Let $\not \partial$ denote the Dirac operator on $M$ and $\tilde{\not}$ its lift to $\tilde{M}$. Then $\tilde{\not}$ descends to a leafwise elliptic operator $\not_{\alpha}$ on $V$ with respect to the foliation $\mathcal{F}$ with leaves the images of $\tilde{M} \times\{g\}$ in $V$.

In this context one can apply the higher index theorem of Connes [4]. It provides a topological formula for the pairing of the index of a leafwise elliptic operator, as an element of K-theory, with a cyclic cocycle in $H^{*}\left(C_{c}^{\infty}\left(U_{n} \rtimes \Gamma\right)\right)$. We will use the form presented by Connes that involves a localization map, $\lambda: H P^{*}\left(C_{c}^{\infty}\left(U_{n} \rtimes \Gamma\right)\right) \rightarrow$ $H^{*+n^{2}}(V)$; see [4], p. 274. Since, in the present context, the normal bundles of all foliations, or the tangent bundles of the units in the étale versions, are oriented we will avoid the use of twisted cohomology groups and always assume that we have identified them with an untwisted group. We will make strong use of the fact that $\phi$, and hence also $\lambda$, are isomorphisms. Suppose also that we are given a vector bundle $E$ on $V$.

There is an invariant transverse measure for the foliation of $V$ and an associated Ruelle-Sullivan current which we will denote by $\Lambda$.

Theorem 6.1 (Connes). Let $\mathrm{Ch}\left(\sigma\left(\not_{\alpha}\right)\right)$ denote the Chern character of the symbol of $\partial_{\alpha}$, and $\operatorname{Td}(T \mathcal{F} \otimes \mathbb{C})$ the Todd class of the complexified tangent bundle along the leaves. Let $\Phi$ be the Thom isomorphism for $T \mathcal{F}$ and let $\operatorname{Ind}\left(\not_{\alpha} \otimes E\right) \in K_{*}\left(C\left(U_{n}\right) \rtimes \Gamma\right)$ be the K-theoretic index of the operator $\not_{\alpha} \otimes E$. Let $[c] \in H^{*}\left(C_{c}^{\infty}\left(U_{n} \rtimes \Gamma\right)\right)$ be given. Then one has

$$
\left\langle\operatorname{Ind}\left(\not \not_{\alpha} \otimes E\right),[c]\right\rangle=\left\langle\lambda(c) \cup \operatorname{Ch}(E) \cup \Phi_{T^{*} \mathcal{F}}^{-1}\left(\operatorname{Ch}\left(\sigma\left(\not \not_{\alpha}\right)\right) \cup \operatorname{Td}(T \mathscr{F} \otimes \mathbb{C}),[\Lambda]\right\rangle .\right.
$$


We next want to obtain a more precise version of the topological pairing in the case that the cyclic class $[c]=\hat{\chi}([u])$, with $[u] \in H^{*}\left(U_{n}\right)$. According to Proposition 5.1 there are invariant polynomials $\mathcal{P}_{1}, \ldots, \mathcal{P}_{i}$ so that $\chi([u])=\hat{\alpha}^{*}\left(T \mathcal{P}_{1} \ldots T \mathcal{P}_{i}\right)$. Thus, (6.1) can be rewritten as

$$
\begin{aligned}
\left\langle\operatorname{Ind}\left(\not \not_{\alpha} \otimes E\right),[c]\right\rangle=\left\langle\hat{\alpha}^{*}\left(T \mathcal{P}_{1} \ldots T \mathcal{P}_{i}\right) \cup p^{*} \operatorname{Ch}(E)\right. & \\
& \cup \Phi_{T^{*} \mathcal{F}}^{-1}(\operatorname{Ch}(\sigma(\not \partial \alpha)) \cup \operatorname{Td}(T \mathscr{F} \otimes \mathbb{C}),[\Lambda]\rangle .
\end{aligned}
$$

If we further assume the existence of a trivialization $\theta: \tilde{M} \times{ }_{\Gamma} U_{n} \rightarrow M \times U_{n}$, then we can simplify to

$$
\begin{aligned}
& \left\langle\operatorname{Ind}\left(\not_{\alpha} \otimes E\right),[c]\right\rangle=\left\langle\hat{\pi}^{*} \hat{\theta}^{*}\left(T \mathcal{P}_{1} \ldots T \mathcal{P}_{i}\right) \cup p^{*} \operatorname{Ch}(E)\right. \\
& \cup \Phi_{T^{*} \mathcal{F}}^{-1}\left(\operatorname{Ch}\left(\sigma\left(\not \partial_{\alpha}\right)\right) \cup \operatorname{Td}(T \mathscr{F} \otimes \mathbb{C}),[\Lambda]\right\rangle .
\end{aligned}
$$

Recall that we defined $T \mathcal{P}(\theta, \alpha)=\hat{\theta}^{*}\left(T \mathcal{P}_{1} \ldots T \mathcal{P}_{i}\right)$. Following the argument in [13], this may be used to obtain the index by a pairing on the base $M$. Thus, we finally obtain the desired formula.

Proposition 6.2. Let $V=\tilde{M} \times_{\alpha} U_{n}$ be the foliated, flat, bundle obtained from the representation $\alpha: \pi_{1}(M) \rightarrow U_{n}$. Let $\theta: \tilde{M} \times_{\alpha} U_{n} \rightarrow M \times U_{n}$ be a trivialization. Let $[c] \in \chi\left(H^{*}\left(U_{n}\right)\right)$ correspond to $T \mathcal{P}_{1} \ldots T \mathcal{P}_{i}$. Then we have

$$
\begin{aligned}
\left\langle\operatorname{Ind}\left(\not_{\alpha} \otimes E\right),[c]\right\rangle=\langle & T \mathcal{P}(\theta, \alpha) \cup \operatorname{Ch}(E) \\
& \cup \Phi_{T^{*} M}^{-1}(\operatorname{Ch}(\sigma(\not \partial)) \cup \operatorname{Td}(T M \otimes \mathbb{C}),[M]\rangle .
\end{aligned}
$$

If we now specialize to the case where the bundle $E$ is the spinors, so that $\not \partial \otimes E$ is the signature operator on $M$, which we denote $D_{M}^{\text {sign }}$, then the operator on $\tilde{M} \times_{\Gamma} U_{n}$ is the leafwise signature operator, and we obtain the following formula, which we will apply in the next section.

\section{Proposition 6.3.}

$$
\left\langle\operatorname{Ind}\left(D_{M, \alpha}^{\mathrm{sign}}\right),[c]\right\rangle=\langle T \mathcal{P}(\theta, \alpha) \cup L(M),[M]\rangle .
$$

\section{Homotopy invariance of characteristic numbers}

In this section we will obtain homotopy invariance results for characteristic numbers obtained from the cyclic classes considered above. We will describe two methods for doing this. The first is based on Connes' method of extending cocycles, cf. [4], [3]. The second was communicated to us by Guoliang Yu, and we will present his argument as an alternative approach. 
7.1. First method. Let $h: M_{1} \rightarrow M_{2}$ be an orientation-preserving homotopy equivalence of closed, oriented manifolds. Let $\alpha_{i}: \Gamma_{i}=\pi_{1}\left(M_{i}\right) \rightarrow U_{n}$ be unitary representations such that $\alpha_{2} h_{*}=\alpha_{1}$.

The first step is to do a suspension operation. For this we lift $h$ to a homotopy equivalence between universal covers, $\tilde{h}: \tilde{M}_{1} \rightarrow \tilde{M}_{2}$, and this descends to a leafwise homotopy equivalence between the foliated, flat, principal bundles:

$$
\hat{h}: V_{1}=\tilde{M}_{1} \times_{\alpha_{1}} U_{n} \rightarrow \tilde{M}_{2} \times_{\alpha_{2}} U_{n}=V_{2}
$$

The signature operators on $M_{i}$ induce operators $D_{M_{i}, \alpha_{i}}^{\text {sign }}$ on $V_{i}$, which are the leafwise signature operators. The map $\hat{h}$ yields a Morita equivalence which induces a map on the K-theory of the associated (étale) foliation algebras,

$$
\left.\left.\hat{h}_{*}: K_{*}\left(C\left(U_{n}\right) \rtimes_{\alpha_{1}} \Gamma_{1}\right)\right) \rightarrow K_{*}\left(C\left(U_{n}\right) \rtimes_{\alpha_{2}} \Gamma_{2}\right)\right),
$$

and it follows from [22], [18] that

$$
\hat{h}_{*}\left(\operatorname{Ind}\left(D_{M_{1}, \alpha_{1}}^{\text {sign }}\right)\right)=\operatorname{Ind}\left(D_{M_{2}, \alpha_{2}}^{\text {sign }}\right) .
$$

Recall that one can always represent the K-theory class of the index in the image of $K_{*}\left(C_{c}^{\infty}\left(\Gamma \ltimes U_{n}\right)\right)$ in such a way that any of our cocycles can pair with it. However, the equivalence in (7.3) is in $K_{*}\left(C\left(U_{n}\right) \rtimes \Gamma\right)$, so there is no guarantee that $\left\langle\hat{h}_{*}\left(\operatorname{Ind}\left(D_{M_{1}, \alpha_{1}}^{\text {sign }}\right)\right),[c]\right\rangle$ will agree with $\left\langle\operatorname{Ind}\left(D_{M_{1}, \alpha_{1}}^{\text {sign }}\right), \hat{h}^{*}([c])\right\rangle$. If the cocycle $[c]$ extends to the crossed-product $\mathrm{C}^{*}$-algebra, then we do obtain equality. Thus, we have the following version of the homotopy invariance property expressed in the Novikov conjecture.

\section{Proposition 7.1.}

$$
\left\langle\operatorname{Ind}\left(D_{M_{2}, \alpha_{2}}^{\text {sign }}\right),[c]\right\rangle=\left\langle\hat{h}_{*}\left(\operatorname{Ind}\left(D_{M_{1}, \alpha_{1}}^{\text {sign }}\right)\right),[c]\right\rangle=\left\langle\operatorname{Ind}\left(D_{M_{1}, \alpha_{1}}^{\text {sign }}\right), \hat{h}^{*}([c])\right\rangle,
$$

where $\hat{h}^{*}$ is the map induced on cyclic theory of the crossed-products.

Now if we interpret the pairings using the results from the previous sections, these equalities can be expressed in terms of characteristic numbers. Suppose that the cyclic class $[c]$ corresponds to a cohomology class in $\chi\left(H^{*}\left(U_{n}\right)\right)$. There is an integer $N$ so that the bundle defined by $\alpha_{1}^{N}$ is trivial, and we will choose an explicit trivialization $\theta$. With this data, we can apply the index formulas (6.3) to (7.4) and obtain homotopy invariance results for certain characteristic numbers.

Theorem 7.2. $\left\langle h^{*}\left(L\left(M_{2}\right)\right) \cup T \mathcal{P}\left(\alpha_{1}^{N}, \theta\right),\left[M_{1}\right]\right\rangle=\left\langle L\left(M_{2}\right) \cup T \mathscr{P}\left(\alpha_{2}^{N}, \theta\right),\left[M_{2}\right]\right\rangle$. 
Proof. By the index theorem, (6.1), the first and third terms in (7.4) can be rewritten in terms of topological pairings with the Ruelle-Sullivan currents associated to the Haar measure, and then, because of the form of $\chi([c])$, the pairing can be done on the base. The resulting formula is as stated.

This should be viewed as a homotopy invariance property for the "local part" of an invariant which is defined in special cases. For the case of Tch, the transgressed Chern character, the invariant is the $\rho$-invariant of Atiyah-Patodi-Singer and we are obtaining $\int_{0}^{1} \dot{\eta}\left(D_{t}\right) d t$, where $D_{t}$ is a path of operators determined by the two flat connections. Homotopy invariance for the $\rho$-invariant itself has been proved under the condition that the Baum-Connes conjecture holds for $C_{\max }^{*}(\Gamma)$. However, it has been shown to hold in $\mathbb{R} / \mathbb{Q}$ in general; see [16], [24], [32].

7.2. Second method. The second approach, communicated to us by Guoliang Yu, does not require the extending of cocycles. We will sketch the argument, which is an adaptation of the usual proof that injectivity of the assembly map implies the Novikov conjecture.

Proposition 7.3. The assembly map

$$
\mu: K^{\mathrm{top}}\left(U_{n}^{\delta} ; C\left(U_{n}\right)\right) \rightarrow K_{*}\left(C\left(U_{n}\right) \rtimes_{\max } U_{n}^{\delta}\right)
$$

is injective. Moreover, there is an injective map

$$
\xi: K_{*}\left(\underline{E U_{n}^{\delta}} \times_{U_{n}^{\delta}} U_{n}\right) \otimes \mathbb{Q}=K_{*}\left(\overline{B U}_{n}\right) \otimes \mathbb{Q} \rightarrow K^{\mathrm{top}}\left(U_{n}^{\delta} ; C\left(U_{n}\right)\right) \otimes \mathbb{Q} .
$$

Assuming for $\left(M_{i}, \alpha_{i}, \theta_{i}\right)$ the same data as in Section 7.1, we get leafwise signature operators and a map $\hat{\theta}_{i}: M_{i} \rightarrow \overline{B U}_{n}$ with the property that $\mu\left(\xi\left[\left(M_{i}, \theta_{i}\right)\right]\right)=$ $\operatorname{Ind}\left(D_{M_{i}, \alpha_{i}}^{\text {sign }}\right)$. According to [18], one has $\operatorname{Ind}\left(D_{M_{2}, \alpha_{2}}^{\text {sign }}\right)=\hat{h}_{*}\left(\operatorname{Ind}\left(D_{M_{1}, \alpha_{1}}^{\text {sign }}\right)\right)$ and since $\mu$ and $\xi$ are injective we have that $\left[\left(M_{2}, \hat{\theta}_{2} h\right)\right]=\left[\left(M_{1}, \hat{\theta}_{1}\right)\right]$. Our goal is to show that for any class $[u] \in H C^{*}\left(\mathscr{H}\left(U_{n}^{\delta} \ltimes U_{n}\right)\right)_{n^{2}}$ we have

$$
\left\langle\operatorname{Ind}\left(D_{M_{2}, \alpha_{2}}^{\text {sign }}\right), \chi([u])\right\rangle=\left\langle\hat{h}_{*}\left(\operatorname{Ind}\left(D_{M_{1}, \alpha_{1}}^{\text {sign }}\right)\right), \chi([u])\right\rangle .
$$

Since the map $\phi: \bigoplus_{k} H^{*+2 k}\left(\overline{B U}_{n}\right) \rightarrow H P^{*-n^{2}}\left(C_{c}^{\infty}\left(U_{n}^{\delta} \ltimes U_{n}\right)\right)$ is an isomorphism, there is a class $x \in H^{*}\left(\overline{B U}_{n}\right)$ satisfying

$$
\left\langle\operatorname{Ch}\left(\left[M_{i}, \theta_{i}\right]\right), x\right\rangle=\left\langle\operatorname{Ind}\left(D_{M_{i}, \alpha_{i}}^{\text {sign }}\right), \chi([u])\right\rangle .
$$

Since the left side is independent of $i$ so is the right, and we obtain

\section{Proposition 7.4.}

$$
\left\langle\operatorname{Ind}\left(D_{M_{2}, \alpha_{2}}^{\text {sign }}\right), \chi([u])\right\rangle=\left\langle\left(\operatorname{Ind}\left(D_{M_{1}, \alpha_{1}}^{\text {sign }}\right)\right), \hat{h}^{*} \chi([u])\right\rangle .
$$


Note that this last step is the reverse of the usual procedure. Here we have a cyclic class and we must find a cohomology class corresponding to it, while in Connes' argument one has a cohomology class and one must find a corresponding cyclic class which also extends to K-theory of the crossed-product.

\section{Concluding remarks}

For a finitely presented group $\Gamma$, let $\operatorname{LP}(\Gamma) \subseteq H^{*}(\Gamma, \mathbb{Q})$ denote the elements obtained by the above process. That is, given a finite dimensional unitary representation of $\Gamma$, we consider $\chi\left(H C^{*}\left(\mathscr{H}\left(U_{n}^{\delta} \ltimes U_{n}\right)_{n^{2}}\right) \subseteq H^{*-n^{2}}\left(\tilde{M} \times_{\Gamma} U_{n}\right)\right.$ and we integrate these classes along the fiber to obtain a subgroup of $H^{*}(B \Gamma)$. By the above arguments, pairing these classes with the index of signature operators gives homotopy invariant characteristic numbers. This is the unitary analog of Connes' work on Gelfand-Fuchs classes (LP stands for Lazarov-Pasternack classes).

\section{References}

[1] J.-L. Brylinski, The degeneracy of two spectral sequences. In The Gelfand Mathematical Seminars, 1990-1992, Birkhäuser, Boston 1993, 1-10. Zbl 0840.55016 MR 1247279

[2] J.-L. Brylinski and V. Nistor, Cyclic cohomology of étale groupoids. K-Theory 8 (1994), 341-365. Zbl 0812.19003 MR 1300545

[3] A. Connes, Cyclic cohomology and the transverse fundamental class of a foliation. In Geometric methods in operator algebras (Kyoto, 1983), Pitman Res. Notes Math. Ser. 123, Longman Sci. Tech., Harlow 1986, 52-144. Zbl 0647.46054 MR 0866491

[4] A. Connes, Noncommutative geometry. Academic Press, San Diego 1994.Zbl 0818.46076 MR 1303779

[5] A. Connes, M. Gromov, and H. Moscovici, Group cohomology with Lipschitz control and higher signatures. Geom. Funct. Anal. 3 (1993), 1-78. Zbl 0789.58069 MR 1204787

[6] A. Connes and D. Kreimer, Hopf algebras, renormalization and noncommutative geometry. Comm. Math. Phys. 199 (1998), 203-242. Zbl 0932.16038 MR 1660199

[7] A. Connes and H. Moscovici, Cyclic cohomology, the Novikov conjecture and hyperbolic groups. Topology 29 (1990), 345-388. Zbl 0759.58047 MR 1066176

[8] A. Connes and H. Moscovici, The local index formula in noncommutative geometry. Geom. Funct. Anal. 5 (1995), 174-243. Zbl 0960.46048 MR 1334867

[9] A. Connes and H. Moscovici, Hopf algebras, cyclic cohomology and the transverse index theorem. Comm. Math. Phys. 198 (1998), 199-246. Zbl 0940.58005 MR 1657389

[10] A. Connes and H. Moscovici, Cyclic cohomology and Hopf algebra symmetry. Lett. Math. Phys. 52 (2000), 1-28. Zbl 0974.58006 MR 1800488 
[11] A. Connes and H. Moscovici, Differentiable cyclic cohomology and Hopf algebraic structures in transverse geometry. In Essays on geometry and related topics, Vol. 1, Monogr. Enseign. Math. 38, Enseignement Math., Geneva 2001, 217-255. Zbl 1018.57013 MR 1929328

[12] M. Crainic, Cyclic cohomology of étale groupoids: the general case. K-Theory 17 (1999), 319-362. Zbl 0937.19007 MR 1706117

[13] R. G. Douglas, S. Hurder, and J. Kaminker, Cyclic cocycles, renormalization and etainvariants. Invent. Math. 103 (1991), 101-179. Zbl 0721.58049 MR 1079841

[14] J. L. Dupont, Simplicial de Rham cohomology and characteristic classes of flat bundles. Topology 15 (1976), 233-245. Zbl 0331.55012 MR 0413122

[15] J. L. Dupont, Characteristic classes for flat bundles and their formulas. Topology 33 (1994), 575-590. Zbl 0860.57020 MR 1286932

[16] M. S. Farber and J. P. Levine, Jumps of the eta-invariant. With an appendix by Shmuel Weinberger: Rationality of $\rho$-invariants. Math. Z. 223 (1996), 197-246, Zbl 0867.57027 MR 1417429

[17] A. Gorokhovsky, Secondary characteristic classes and cyclic cohomology of Hopf algebras. Topology 41 (2002), 993-1016. Zbl 1008.58008 MR 1923996

[18] M. Hilsum and G. Skandalis, Invariance par homotopie de la signature à coefficients dans un fibré presque plat. J. Reine Angew. Math. 423 (1992), 73-99. Zbl 0731.55013 MR 1142484

[19] X. Jiang, An index theorem on foliated flat bundles. K-Theory 12 (1997), 319-359. Zbl 0913.58054 MR 1485433

[20] J. D. S. Jones and B. W. Westbury, Algebraic $K$-theory, homology spheres, and the $\eta$ invariant. Topology 34 (1995), 929-957. Zbl 0867.55006 MR 1362792

[21] J. Kaminker, Algebraic $K$-theory invariants for operator theory. In Multivariable operator theory (Seattle, 1993), Contemp. Math. 185, Amer. Math. Soc., Providence, RI, 1995, 187-194. Zbl 0831.46080 MR 1332060

[22] J. Kaminker and J. G. Miller, Homotopy invariance of the analytic index of signature operators over $C^{*}$-algebras. J. Operator Theory 14 (1985), 113-127. Zbl 0614.46062 MR 789380

[23] M. Karoubi, Homologie cyclique et $K$-théorie. Astérisque 149 (1987). Zbl 0648.18008 MR 913964

[24] N. Keswani, Homotopy invariance of relative eta-invariants and $C^{*}$-algebra $K$-theory. Electron. Res. Announc. Amer. Math. Soc. 4 (1998), 18-26. Zbl 0889.19003 MR 1613055

[25] M. Khalkhali and B. Rangipour, Para-Hopf algebroids and their cyclic cohomology. Lett. Math. Phys. 70 (2004), 259-272. Zbl 1067.58007 MR 2128954

[26] C. Lazarov and J. Pasternack, Secondary characteristic classes for Riemannian foliations. J. Differential Geometry 11 (1976), 365-385. Zbl 0356.57007 MR 0445513

[27] J.-L. Loday, Cyclic homology. 2nd ed., Grundlehren Math. Wiss. 301. Springer-Verlag, Berlin 1998. Zbl 0885.18007 MR 1600246 
[28] J.-H. Lu, Hopf algebroids and quantum groupoids. Internat. J. Math. 7 (1996), 47-70. Zbl 0884.17010 MR 1369905

[29] I. Moerdijk, Proof of a conjecture of A. Haefliger. Topology 37 (1998), 735-741. Zbl 0897.22003 MR 1607724

[30] H. Moriyoshi and T. Natsume, The Godbillon-Vey cyclic cocycle and longitudinal Dirac operators. Pacific J. Math. 172 (1996), 483-539. Zbl 0852.58077 MR 1386629

[31] J. Mrčun, The Hopf algebroids of functions on étale groupoids and their principal Morita equivalence. J. Pure Appl. Algebra 160 (2001), 249-262; erratum ibid. 176 (2002), 275-276. Zbl 0986.16017 MR 1836002 MR 1933720

[32] P. Piazza and T. Schick, Bordism, rho-invariants and the Baum-Connes conjecture. J. Noncommut. Geom. 1 (2007), 27-111. Zbl 05349402 MR 2294190

Received December 21, 2007

J. Kaminker, Department of Mathematics, IUPUI, Indianapolis, IN 46202, and Department of Mathematics, UC Davis, Davis, CA 95616 U.S.A.

E-mail: kaminker@math.ucdavis.edu

X. Tang, Department of Mathematics, Washington University, Campus Box 1146, St. Louis, MO, 63130-4899, U.S.A.

E-mail: xtang@math.wustl.edu 